\title{
Generation of a Train of Ultrashort Pulses using Periodic Waves in Tapered Photonic Crystal Fibres
}

\author{
S. O. Atuba ${ }^{\mathrm{a}}$, K. Nakkeeran ${ }^{\mathrm{a}}$, K. W. Chow ${ }^{\mathrm{b}}$, P. Ramesh Babu ${ }^{\mathrm{c}}$, A. Manimegalai ${ }^{\mathrm{d}}$ and K. Senthilnathan ${ }^{\mathrm{c} *}$ \\ ${ }^{a}$ School of Engineering, Fraser Noble Building, University of Aberdeen, Aberdeen AB24 3UE, UK; \\ ${ }^{b}$ Department of Mechanical Engineering, University of Hong Kong, Pokfulam Road, Hong Kong; \\ ${ }^{c}$ Photonics, Nuclear and Medical Physics Division, School of Advanced Sciences, VIT University, Vellore, \\ India \\ ${ }^{d}$ School of Electronics Engineering, VIT University, Vellore, India
}

(Received 00 Month 20XX; final version received 00 Month 20XX)

\begin{abstract}
We consider a light wave propagation in tapered photonic crystal fibres (PCFs) wherein the wave propagation is described by the variable coefficient nonlinear Schrödinger equation (NLSE). We solve it directly by means of the theta function identities and Hirota bilinear method in order to obtain the exact periodic waves of sn, cn and dn types. These chirped period waves demand exponential variations in both dispersion and nonlinearity. Besides, we analytically demonstrate the generation of a train of ultrashort pulses using the periodic waves by exploiting the exponentially varying optical properties of the tapered PCFs. As a special case, we discuss the chirped solitary pulses under long wave limit of these periodic waves. In addition, we derive these types of periodic waves using the self-similar analysis and compare the results.
\end{abstract}

Keywords: nonlinear optics; photonic crystal fiber; pulse compression

\section{Introduction}

The dynamical balance experienced by group velocity dispersion (GVD) with the self-phase modulation (SPM), a nonlinear effect, is regarded to be a notable achievement in soliton communication systems since these effects, respectively, broaden the pulse in both temporal and spectral domains $[1,2]$. With this stabilization resulting from the combined effects of the SPM and GVD, the optical solitons can travel over a long distance without considerable loss and shape change. The nonlinear Schrödinger equation (NLSE) was modeled to describe the propagation of optical pulses in a high speed, long-distance optical fibre system [1, 2]. Later, Mollenauer and his co-workers demonstrated the generation of optical solitons experimentally in optical fibres [3, 4].

Recently, high repetition-rate ultrashort pulses (USPs) have found wide applications, especially in optical coherence tomography, material processing, and instrumentation [5]. A pulse train of femtosecond range with a repetition rate as high as $1 \mathrm{GHz}$ has been produced by passive mode locked lasers [5]. Although by using active mode locked lasers, it is possible to produce a pulse train with a high repetition rate exceeding $40 \mathrm{GHz}$, it still continues to be a challenging issue owing to the presence of modulator within the cavity of such laser systems. Besides, the existing opto-electronic direct modulation techniques cannot cope with such high bit rates. As an alternative idea, the nonlinear conversion of a dual-frequency optical signal through modulational instability was proposed [6]. A train of high-energy pulses with adjustable separations has been produced using

${ }^{*}$ Corresponding author. Email: Senthee@gmail.com 
Michelson interferometers [7]. But this is proven to be impractical for operations involving more number of pulses [8]. Eventually, pulse compression turns out to be one of the best techniques for generating USPs [5]. The most successful fibre based pulse compression schemes are fibre-grating compression, soliton-effect compression and adiabatic soliton compression [5, 9-13].

The NLSE has been found to be an adequate model to describe the evolution of the nonlinear envelope pulses in the ultrashort pulse regime (from ps to sub-ps). The time domain approach is widely used method to solve the NLSE directly [14]. By numerically solving NLSE with input being continuous wave $(\mathrm{CW})$, it has been shown that the $\mathrm{CW}$ beam evolves into a narrow pulse train [6]. The fibre length needed to produce this train of narrow pulses relies on the initial modulation depth. On further propagation, the multipeak structure cripples out and subsequently returns to the original form [15]. This characteristic is generic when the NLSE is solved by taking steady state of its arbitrary periodic modulation [16]. This suggests that the NLSE should possess the periodic solutions whose particular form changes with propagation. These periodic solutions can be expressed in their generic and compact form as Jacobian elliptic functions [15]. The present NLSE model differs from other similar variable coefficient NLSE as the inhomogeneous parameters involve both spatial and time coordinates [17]. Several research groups have successfully employed the inverse scattering transform as well as Bäcklund transform to obtain the exact solutions for completely integrable variable coefficient [18-22]. In this paper, the Hirota bilinear transformation (HBT), a simple but powerful method, is deployed to obtain a set of new periodic wave solutions for variable coefficient NLSE system. Its uniqueness lies in the fact that periodic and solitary wave solutions are obtained even for non-integrable systems [23, 24]. Besides, we make use of these periodic waves and compress them into a train of USPs by exploiting the optical properties of tapered PCFs.

This paper is presented as follows. In Section 2, we discuss the required theoretical model wherein we delve into the light propagation in tapered PCFs using NLSE. In Section 3, we solve the NLSE using HBT method and discuss the generation of periodic waves of sn, cn and dn types in the exponentially decreasing dispersion and nonlinearity increasing tapered PCF. Further, in Section 4 , we explore the different combinations of exponential variations of dispersion and nonlinearity for the existence of above mentioned chirped periodic waves. In Section 5, based on these analytical results, we design the required tapered PCFs for achieving the various exponential variations. Then, by exploiting these optical properties, we discuss the generation of a train of USPs. Further, we present the solitary type pulses in the long wave limit in Section 6. In Section 7, we delineate the generation of chirped periodic waves using the self-similar analysis and compare the results with the HBT method. Finally, the conclusion of the research findings is presented in Section 8.

\section{Light Propagation in Tapered Photonic Crystal Fibres}

The wave propagation in tapered PCFs is governed by the following NLSE and is given by

$$
i \frac{\partial A}{\partial z}+p(z) \frac{\partial^{2} A}{\partial t^{2}}+q(z)|A|^{2} A+i g(z) A=0 .
$$

Here, $A$ is the axial electric field of the complex envelope. The distance is denoted as $z$ and the retarded time as $t$. Further, $p(z), q(z)$ and $g(z)$ represent the dispersion, nonlinearity and linear gain/loss, respectively [15]. This NLSE model not only applicable in optical fibres, but also in Bose-Einstein condensation (BEC), superconductivity, superfluidity, etc [25]. The coefficients in Eq. (1) represent the different physical situations and their corresponding applications in various domains. Recently, the periodic waves have been studied when $p$ is real and $q$ is complex [24].

Various exact solutions, namely, holes, fronts, bright and dark solitons for complex case of $p$ and $q$ have been reported using a modified form of the basic Hirota method [26]. A family of variable coefficient NLSEs has been solved exactly by means of bilinear approach which is an extension 
of the basic Hirota method. It has been shown by inverse scattering transform that the variable coefficient NLS Eq. (1) is integrable [17, 27]. Further, this equation can be mapped into a constant coefficient NLS type equation and the details can be found in $[28]$.

\section{Chirped Periodic Waves using Hirota Bilinear Method}

The procedure for solving such special evolution equations with variable coefficients includes two basic steps: (a) First, an appropriate chirp factor is factorized out, and (b) Hirota method with space-(or time-) dependent wave numbers is applied. The Hirota method is also used to handle the variable coefficient NLSE with nonlinear gain/loss. In this paper, we discuss the various types of periodic waves and solitary type pulses in the long wave limit by analytically solving Eq. (1) using HBT technique. The modified Hirota method has the following key features. First, the pulse chirp is accounted for by adding the chirp factor,

$$
A=\exp \left(\frac{i \beta t^{2}}{2}\right) \psi, \quad \beta=\beta(z) .
$$

We substitute Eq. (2) in Eq. (1) and identify the following condition for $\beta(z)$,

$$
\frac{\beta_{z}}{2}+p \beta^{2}=0
$$

where, $\beta_{z}$ denotes the partial derivative with respect to $z$. Since the dispersion $p$ is real, the expression for function $\psi$ from Eq. (2) is

$$
i \psi_{z}+p \psi_{t t}+2 i \beta p t \psi_{t}+i \beta p \psi+q|\psi|^{2} \psi+i \gamma \psi=0 .
$$

Light waves of special modes can be obtained by expressing the wave patterns as

$$
\psi=\frac{g \exp \left(-i \Omega_{0}\right)}{f} .
$$

The constraint here is that $f$ is real and for the bilinear process, both $g$ and $f$ are dependent variables. Typically exponential functions are chosen to derive the solitary pulses. On the other hand, elliptic functions are to be chosen for deriving the periodic patterns. The phase factor $\Omega_{0}$ is a function depending on the space only. It has its derivatives determined in the bilinear equation, and hence is easily regained by quadrature. The bilinear expressions are then,

$$
\begin{gathered}
\left(i D_{z}+p D_{t}^{2}+2 i \beta p t D_{t}+\frac{\partial \Omega_{0}}{\partial z}+i \beta p+i \gamma-C\right) g \bullet f \\
\left(p D_{t}^{2}-C\right) f \bullet f=q g g^{*}
\end{gathered}
$$

where $D$ is the Hirota bilinear operator and $C$ is spatially dependent in the case of dark soliton. When it operates on a set of two functions $a(x)$ and $b(x)$, it is defined by [23],

$$
\left.D_{x}^{n} a \bullet b \equiv\left(\frac{\partial}{\partial x}-\frac{\partial}{\partial y}\right)^{n} a(x) b(y)\right|_{y=x}=\left.\frac{\partial^{n}}{\partial y^{n}} a(x+y) b(x-y)\right|_{y=0}
$$


To realise periodic waves of various types, we now work with theta functions [14, 26]. The dispersion and nonlinearity profiles are given by,

$$
\begin{gathered}
p(z)=p_{0} \exp (-\sigma z), \\
q(z)=q_{0} \exp (2 \gamma z),
\end{gathered}
$$

where $\sigma$ and $\gamma$ are real parameters. Eq. (1) now becomes

$$
i \frac{\partial A}{\partial z}+p_{0} e^{-\sigma z} \frac{\partial^{2} A}{\partial t^{2}}+q_{0} e^{2 \gamma z}|A|^{2} A+i \gamma A=0 .
$$

To get a dn-wave, $g$ and $f$ are chosen as theta functions given by [14, 29],

$$
g=A_{0} \theta_{3}\left(t\left[h_{1}(z)\right]\right), \quad f=\theta_{4}\left(t\left[h_{1}(z)\right]\right) .
$$

Here, $A_{0}$, the amplitude parameter is spatial dependent and it has to match the loss/gain factor. Combining Eq. (6) and Eq. (11) gives,

$$
\begin{aligned}
& \theta_{4}\left\{i\left(A_{0} e^{-i \Omega_{0}}\right)_{z} \theta_{3} \theta_{4}+i A_{0} e^{-i \Omega_{0}} D_{z}\left(\theta_{3} \bullet \theta_{4}\right)\right. \\
& +p_{0} e^{-\sigma z} A_{0} e^{-i \Omega_{0}} D_{t}^{2}\left(\theta_{3} \bullet \theta_{4}\right)+2 i \beta p_{0} e^{-\sigma z} t A_{0} e^{-i \Omega_{0}} \bullet \\
& \left.D_{t}\left(\theta_{3} \bullet \theta_{4}\right)+i\left(\beta p_{0} e^{-\sigma z}+\gamma\right) A_{0} e^{-i \Omega_{0}} \theta_{3} \theta_{4}\right\} \\
& +A_{0} e^{-i \Omega_{0}} \theta_{3}\left[-p_{0} e^{-\sigma z} D_{t}^{2}\left(\theta_{4} \bullet \theta_{4}\right)+q_{0} e^{2 \gamma z} A_{0}^{2} \theta_{3}^{2}\right] \\
& =0 .
\end{aligned}
$$

The wave number, $h_{1}(z)$ is also spatial dependent. Its exact expression is realised by equating the odd order Hirota derivatives in Eq. (12) to zero. Hence,

$$
h_{1}=h_{00} e^{\sigma z}
$$

Solving the real terms of Eq. (12) gives

$$
\begin{array}{r}
\theta_{4}\left[A_{0} \Omega_{0 z} \theta_{3} \theta_{4} e^{-i \Omega_{0}}+p A_{0} e^{-i \Omega_{0}} D_{t}^{2}\left(\theta_{3} \bullet \theta_{4}\right)\right]+ \\
A_{0} e^{-i \Omega_{0}} \theta_{3}\left[-p D_{t}^{2}\left(\theta_{4} \bullet \theta_{4}\right)+q A_{0}^{2} \theta_{3}^{2}\right]=0 .
\end{array}
$$

Upon introducing theta identities, we get,

$$
\frac{\partial \Omega_{0}}{\partial z}+p\left(2-k^{2}\right) e^{2 \sigma z}=0
$$

For the dispersion decreasing case, we substitute Eq. (8) in Eq. (15). The resulting solution is,

$$
\Omega_{0}=\frac{-p_{0} e^{\sigma z}\left(2-k^{2}\right)}{\sigma}
$$

Solving the imaginary terms in Eq. (12) and substituting Eq. (8) in Eq. (15), we have,

$$
A_{0}=\eta \exp \left[-\left(\gamma-\frac{\sigma}{2}\right) z\right]
$$


Here, $\eta$ is a constant. Here, we adopt the theta identities [14, 29]. Substituting Eq. (17) in Eq. (12) and equating the like terms results in the following expression for nonlinear coefficient in the case of decreasing dispersion:

$$
q=\frac{2 p_{0} h_{00}^{2} \theta_{3}^{2}(0) \theta_{4}^{2}(0)}{\eta^{2}} \exp (2 \gamma z)
$$

Theta identities are used in obtaining this expression for $q$. For increasing nonlinearity, by using Eq. (9), Eq. (17) takes the form

$$
A_{0}=\left(\frac{2 p_{0} h_{00}^{2} \theta_{3}^{2}(0) \theta_{4}^{2}(0)}{q_{0}}\right)^{\frac{1}{2}} \exp \left[-\left(\gamma-\frac{\sigma}{2}\right) z\right] .
$$

We substitute the above equations in Eq. (2) and introduce the elliptic function identites for generating the periodic waves. The periodic wave of $d n$ type is thus derived as,

$$
A=\sqrt{\frac{2 p_{0}}{q_{0}}} d n[t \exp (\sigma z)] \exp \left\{\left(\frac{\sigma}{2}-\gamma\right) z+\frac{i p_{0} e^{\sigma z}\left(2-k^{2}\right)}{\sigma}-\frac{i \sigma e^{\sigma z} t^{2}}{4 p_{0}}\right\}
$$

where $k$ represents the elliptic modulus function. Here, $p_{0}$ and $q_{0}$ are the dispersion and nonlinearity parameters, respectively, at $z=0$. Similarly, the periodic wave expressed in terms of $c n$ is derived as,

$$
A=k \sqrt{\frac{2 p_{0}}{q_{0}}} c n[t \exp (\sigma z)] \exp \left\{\left(\frac{\sigma}{2}-\gamma\right) z+\frac{i p_{0} e^{\sigma z}\left(2 k^{2}-1\right)}{\sigma}-\frac{i \sigma e^{\sigma z} t^{2}}{4 p_{0}}\right\}
$$

Further, we find another type of periodic wave in terms of $s n$ as,

$$
A=k \sqrt{\frac{2 p_{0}}{q_{0}}} \operatorname{sn}[t \exp (\sigma z)] \exp \left\{\left(\frac{\sigma}{2}-\gamma\right) z-\frac{i p_{0} e^{\sigma z}\left(1+k^{2}\right)}{\sigma}-\frac{i \sigma e^{\sigma z} t^{2}}{4 p_{0}}\right\} .
$$

The exact solutions Eqs. (20), (21) and (22) are called chirped periodic waves. In contrast to the conventional periodic waves, the above mentioned periodic waves possess a linear chirp. These chirped periodic waves facilitate two-stage dynamics of wave evolution. In the initial stage of evolution, linear effect is predominant compared to the nonlinearity. Hence this process results in quasi-linear compression. On the other hand, in the final stage, the nonlinear effect turns much pronounced compared to the linear effect. Therefore, these chirped periodic waves do undergo nonlinear compression.

It may be recalled that there exists a physical constraint that does not allow the simultaneous variation of all the three distributed parameters, namely, dispersion, nonlinearity and gain/loss. Nonetheless, it is possible to vary any two of the three parameters by keeping the third one a constant. Hence, it is necessary to identify the most physically valid system wherein the dispersion and the nonlinearity vary along the propagation direction with the loss remaining a constant. In addition, there is another physical constraint which dictates that the loss coefficient is the same as the exponential growth rate of the nonlinearity i.e., $g(z)=\gamma$. Thus, these crucial physical conditions have to be satisfied for the formation of the chirped periodic waves in the PCFs.

\section{Existence of Periodic Waves for Various Physical Conditions}

In this section, we explore if there are any other possibilities of generating the chirped periodic waves for various choices of positive and negative exponentially varying dispersion and nonlinearity. 
The following are the possible exponential variations.

(1) GVD decreases and nonlinearity increases:

$$
\begin{aligned}
& p(z)=p_{0} \exp (-\sigma z) \\
& q(z)=q_{0} \exp (2 \gamma z)
\end{aligned}
$$

(2) Both GVD and nonlinearity decrease:

$$
\begin{aligned}
& p(z)=p_{0} \exp (-\sigma z) \\
& q(z)=q_{0} \exp (-2 \gamma z)
\end{aligned}
$$

(3) GVD increases and nonlinearity decreases:

$$
\begin{aligned}
& p(z)=p_{0} \exp (\sigma z) \\
& q(z)=q_{0} \exp (-2 \gamma z)
\end{aligned}
$$

(4) Both GVD and nonlinearity increase:

$$
\begin{aligned}
& p(z)=p_{0} \exp (\sigma z) \\
& q(z)=q_{0} \exp (2 \gamma z)
\end{aligned}
$$

These physically realistic conditions are not only merely mathematical conditions for the existence of chirped periodic waves but also they represent the different fiber media. Therefore, we design four distinct PCFs that satisfy their corresponding mathematical conditions. In what follows, we investigate the evolutions of these chirped periodic waves by suitably designing various tapered PCFs.

\section{Designing Tapered PCFs through HBT Conditions}

In this section, we explore the various designs of PCFs by suitably varying the design parameters, namely, the pitch and relative air-hole diameter. We infer that the pitch and the diameter of air holes have to be varied exponentially by tapering the PCFs in order to meet the above mentioned conditions. We deal with the four designs of PCFs in what follows.

\section{Case I: GVD Decreases and Nonlinearity Increases}

First, on the basis of the analytical results, we design a PCF at $1550 \mathrm{~nm}$ with an exponentially decreasing dispersion profile as well as exponentially increasing nonlinearity profile. Here, we decrease the diameter of air holes and the pitches exponentially when the PCF is tapered. To look for a train of USPs at $1550 \mathrm{~nm}$, we design the PCF with large dispersion to realize a compact compressor for which the dispersion length $L_{D}\left(=\tau_{0}^{2} / p_{0}\right)$ becomes smaller. Here, $\tau_{0}$ and $p_{0}$ represent pulse width and dispersion, respectively. Fig. 1 illustrates the geometrical structure of the proposed tapered PCF. The mode field distribution of this fibre is depicted in Fig. 2. We find the initial GVD as $-55.37 \mathrm{ps}^{2} / \mathrm{km}$ by adopting finite element method with the PCF parameters of $\mathrm{d} / \Lambda=0.4$ and $\Lambda=3.35 \mu \mathrm{m}$. Next, we keep the PCF of length $7.44 \mathrm{~m}$, a value two times the dispersion length $\left(\mathrm{L}=2 L_{D}\right)$ where $L_{D}$ is $3.72 \mathrm{~m}$ for a pulse width of $0.8 \mathrm{ps}$. Here, the relative air hole diameter, $\mathrm{d} / \Lambda$, is varied from 0.4 to 0.399352 and the $\Lambda$ from 3.35490 to $3.35373 \mu \mathrm{m}$.

Fig. 3a shows the variation of design parameters, namely, relative air hole diameter and pitch with respect to propagation distance, $z$. The variations of GVD and nonlinearity against distance are illustrated in Fig. 3b. The cumulative dispersion, $p(z)$, at the end of the PCF is computed to 


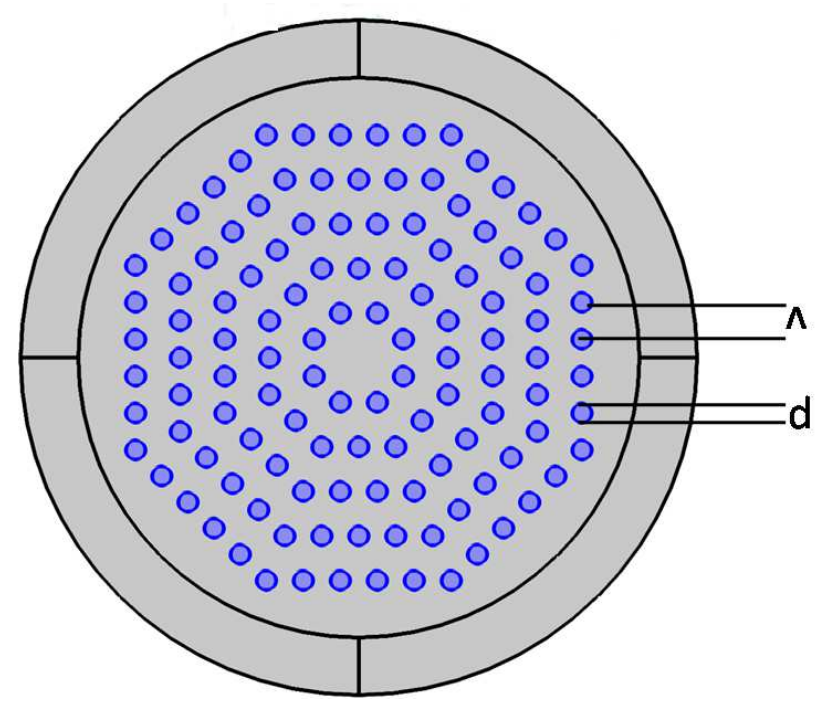

Figure 1.: Structure of the proposed single mode tapered octagonal PCF with $d / \Lambda=0.4$ and $\Lambda=$ $3.35 \mu \mathrm{m}$.

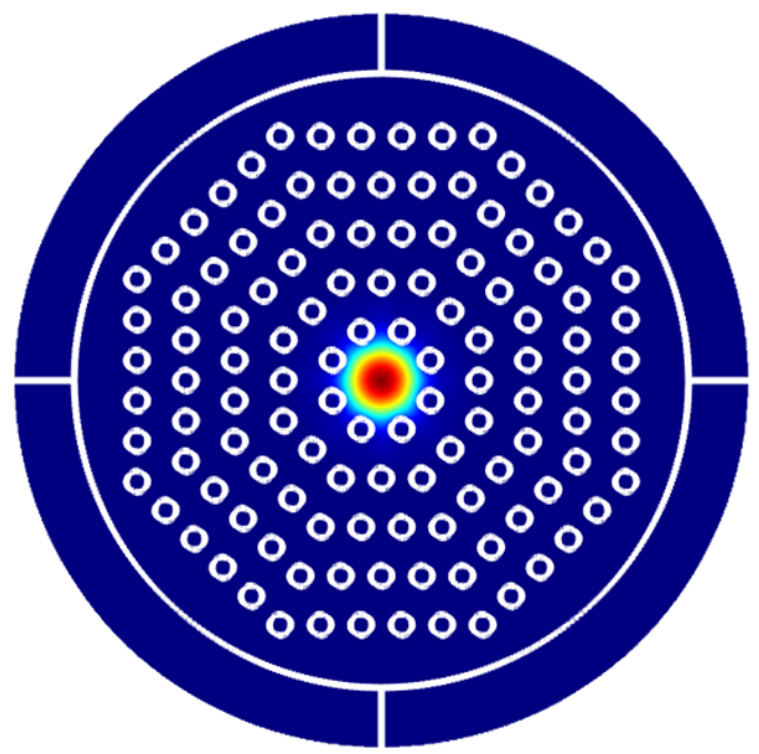

Figure 2.: Mode field distribution of the proposed single mode tapered octagonal PCF with $d / \Lambda=$ 0.4 and $\Lambda=3.35 \mu \mathrm{m}$.

be $-43.21 \mathrm{ps}^{2} / \mathrm{km}$. Next, we calculate the effective nonlinearity using effective mode area, $A_{\text {eff }}$. The nonlinearity in this tapered PCF increases from 6.79 to $7.34 \mathrm{~W}^{-1} \mathrm{~km}^{-1}$.

Having designed the desired tapered PCF of exponentially decreasing dispersion and exponentially increasing nonlinearity profiles, we proceed to investigate the generation of a train of USPs using various periodic waves of $\mathrm{sn}, \mathrm{cn}$ and dn discussed above. In order to understand the dynamics of the chirped periodic waves in the tapered PCF, it is essential to compute the important physical parameters of these waves, namely, amplitude, pulse width and chirp. In a tapered PCF with exponentially decreasing dispersion and exponentially increasing nonlinearity, the amplitude, $A$, and 
(a)

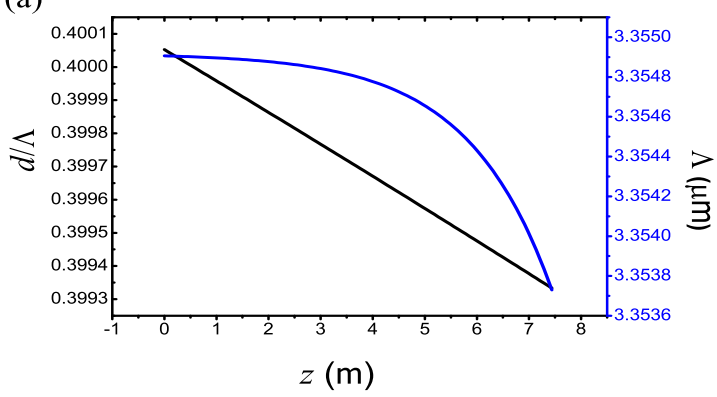

(b)

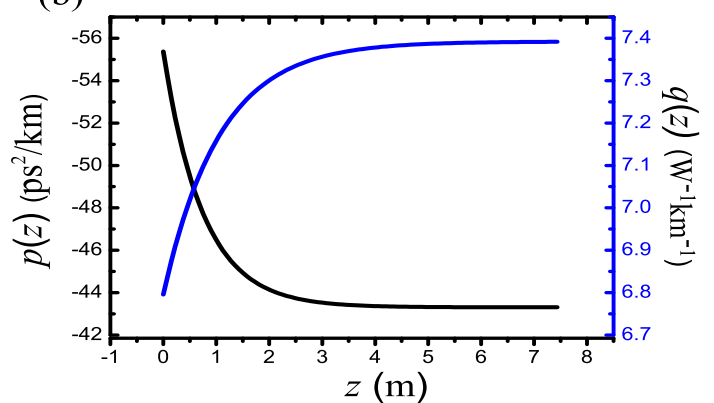

Figure 3.: (a) Variation of PCF design parameters $d / \Lambda$ and $\Lambda$ along the propagation distance, $z$. (b) Variation of GVD, $p(z)$, and nonlinearity, $q(z)$, as a function of propagation distance, $z$.

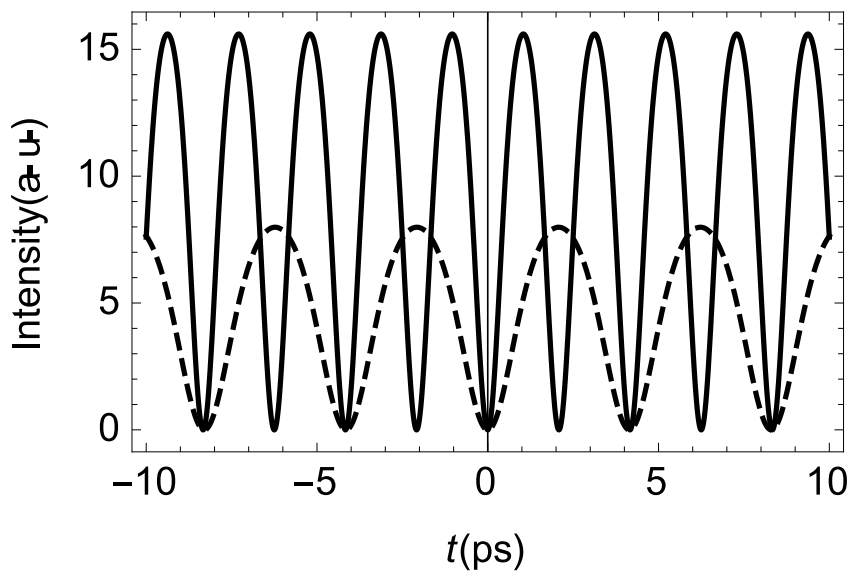

Figure 4.: Intensity profile of initial (dashed lines) and compressed output (solid lines) of the snperiodic wave through a PCF. The physical parameters are $p_{0}=-0.0553713 \mathrm{ps}^{2} / \mathrm{m}, q_{0}=0.006794$ $\mathrm{W}^{-1} \mathrm{~m}^{-1}, \gamma=0.00132022 \mathrm{~m}^{-1}, \sigma=0.092686 \mathrm{~m}^{-1}, L_{D}=3.7187 \mathrm{~m}$ and $k=0.7$.

pulse width, $\tau$, of these chirped periodic waves are given by

$$
\begin{aligned}
A & =k \sqrt{\frac{2 p_{0}}{q_{0}}} \frac{1}{\tau_{0}} \exp \left[\left(\frac{\sigma}{2}-\gamma\right) z\right] \\
\tau & =\tau_{0} \exp (-\sigma z),
\end{aligned}
$$

where $k$ represents the modulus of the elliptic function. From the above expressions, it is obvious that the intensity and chirp increase exponentially while the pulse width decreases exponentially. These physical parameters of the chirped waves signify compression during the course of propagation in this tapered PCF. At this juncture, it is necessary to compute the width of the compressed pulses in order to determine the compression factor (CF) which is defined by the ratio between input pulse width and output pulse width, i.e., $\mathrm{CF}=\frac{\tau_{0}}{\tau(z)}=\exp (\sigma z)$. Thus, we find that the compression factor increases exponentially. Further, we also emphasize that the the decay rate, $\sigma$, of the dispersion influences the self-similar pulse compression process. Besdies, one can also optimize the length of the compressor with the help of following relation, $z=\frac{1}{\sigma} \ln (C F)$. Figs. 4,5 and 6 depict the evolution of the periodic waves of sn, cn and dn types, respectively. In these figures, the periodic waves depicted as dashed lines represent the intensity of the input chirped waves and the waves in solid lines imply the intensity of the compressed pulses. Thus, one can achieve a train of USPs using the tapered PCF for all the types of periodic waves.

Case II: Both GVD and Nonlinearity Decrease

Next, we design a PCF to meet the requirements of both decreasing dispersion and decreasing 


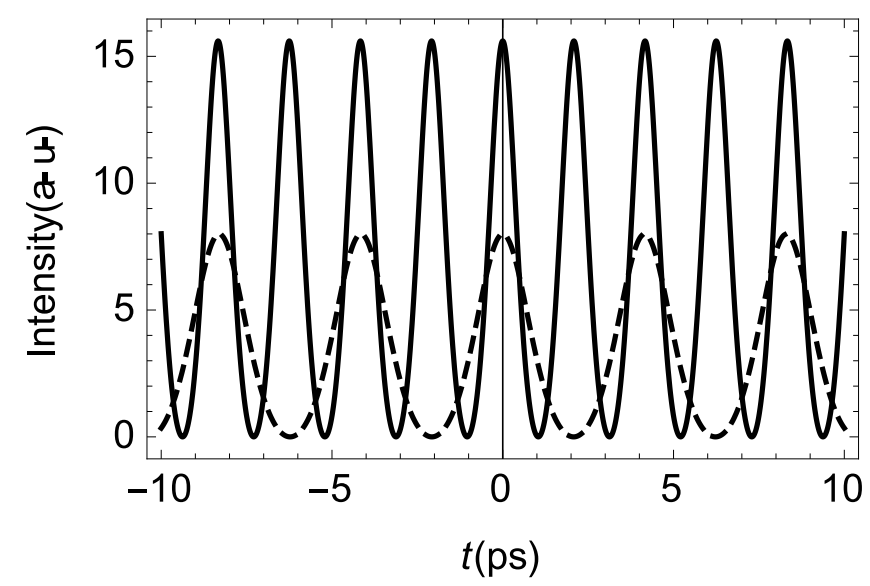

Figure 5.: Intensity profile of initial (dashed lines) and compressed output (solid lines) of the cnperiodic wave through a PCF. The physical parameters are $p_{0}=-0.0553713 \mathrm{ps}^{2} / \mathrm{m}, q_{0}=0.006794$ $\mathrm{W}^{-1} \mathrm{~m}^{-1}, \gamma=0.00132022 \mathrm{~m}^{-1}, \sigma=0.092686 \mathrm{~m}^{-1}, L_{D}=3.7187 \mathrm{~m}$ and $k=0.7$.

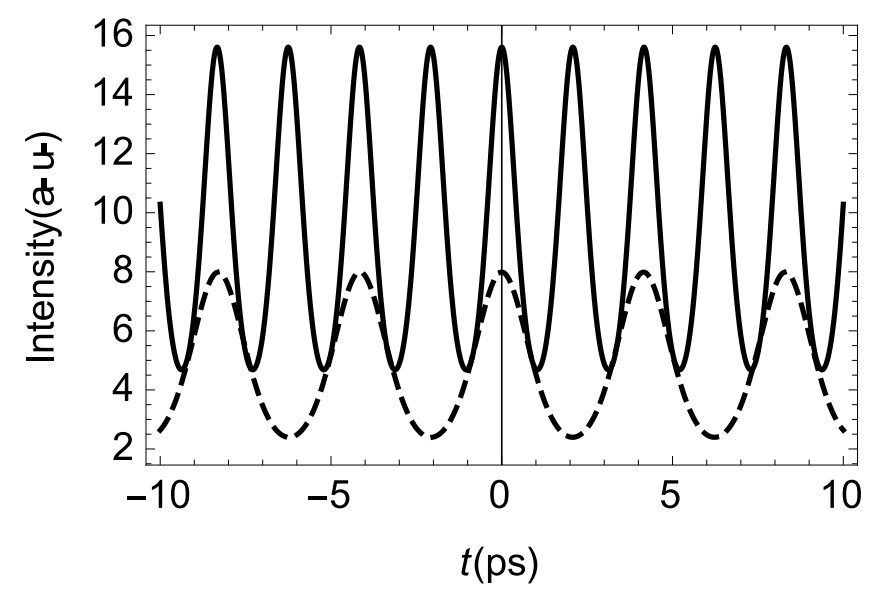

Figure 6.: Intensity profile of initial (dashed lines) and compressed output (solid lines) of the dnperiodic wave through a PCF. The physical parameters are $p_{0}=-0.0553713 \mathrm{ps}^{2} / \mathrm{m}, q_{0}=0.006794$ $\mathrm{W}^{-1} \mathrm{~m}^{-1}, \gamma=0.00132022 \mathrm{~m}^{-1}, \sigma=0.092686 \mathrm{~m}^{-1}, L_{D}=3.7187 \mathrm{~m}$ and $k=0.7$.

nonlinearity to satisfy the physical constraints of Eq. (24). Here, we reduce the diameter of air hole without varying the pitch. We choose a PCF of length $7.44 \mathrm{~m}$ as in the previous case. The relative air hole diameter is kept at 0.4 and the diameters of the air holes vary from 1.342 to 2.8 $\mu \mathrm{m}$ and the pitch is kept at $3.35 \mu \mathrm{m}$. Figs. $7 \mathrm{a}$ and $7 \mathrm{~b}$ show the variations of design parameters and computed optical properties with respect to the propagation distance.

With these designs of PCF, we theoretically demonstrate the generation of a train of USPs for the three types of chirped periodic waves. The $\mathrm{dn}$, cn and sn waves for this case are,

$$
\begin{gathered}
A=\sqrt{\frac{2 p_{0}}{q_{0}}} d n[t \exp (\sigma z)] \exp \left\{\left(\frac{\sigma}{2}+\gamma\right) z+\frac{i p_{0} e^{\sigma z}\left(2-k^{2}\right)}{\sigma}-\frac{i \sigma e^{\sigma z} t^{2}}{4 p_{0}}\right\}, \\
A=k \sqrt{\frac{2 p_{0}}{q_{0}}} \operatorname{cn}[t \exp (\sigma z)] \exp \left\{\left(\frac{\sigma}{2}+\gamma\right) z+\frac{i p_{0} e^{\sigma z}\left(2 k^{2}-1\right)}{\sigma}-\frac{i \sigma e^{\sigma z} t^{2}}{4 p_{0}}\right\},
\end{gathered}
$$



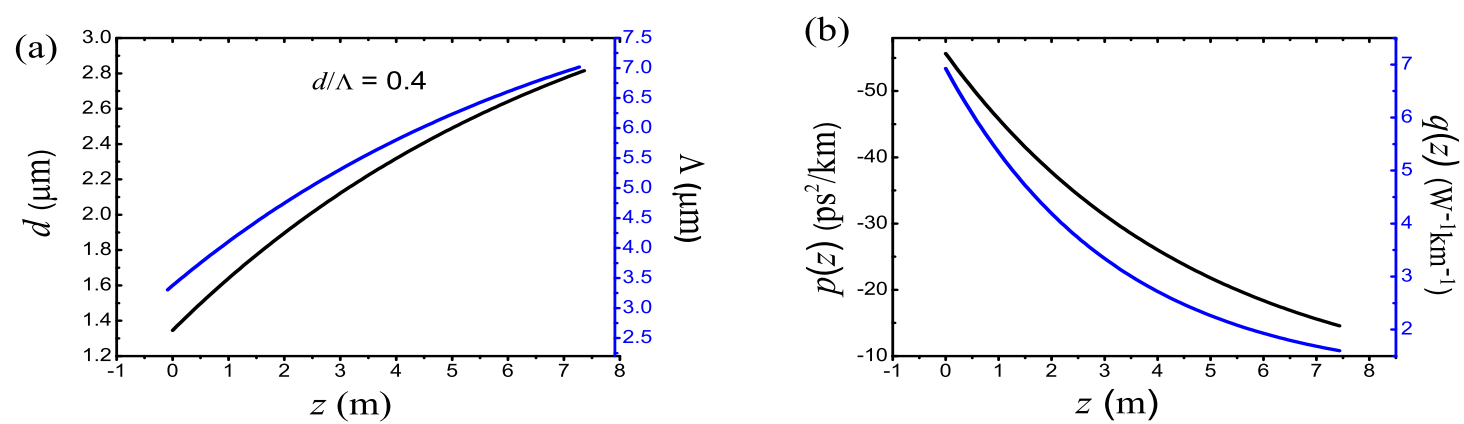

Figure 7.: (a) Variation of PCF design parameters $d$ and $\Lambda$ along the propagation distance, $z$. (b) Variation of GVD, $p(z)$, and nonlinearity, $q(z)$, as a function of propagation distance, $z$.

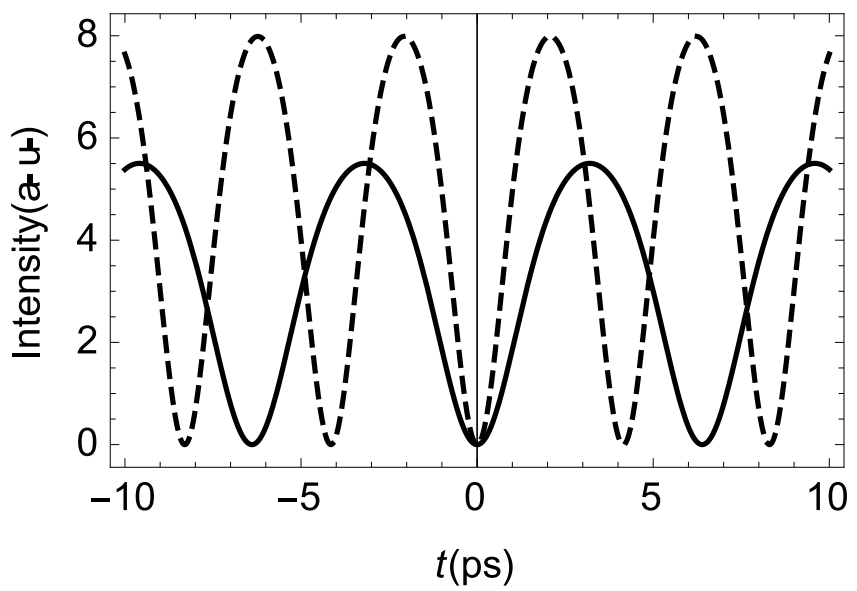

Figure 8.: Intensity profile of initial (dashed lines) and compressed output (solid lines) of the snperiodic wave through a PCF. The physical parameters are $p_{0}=-0.0553713 \mathrm{ps}^{2} / \mathrm{m}, q_{0}=0.006794$ $\mathrm{W}^{-1} \mathrm{~m}^{-1}, \gamma=0.00398355 \mathrm{~m}^{-1}, \sigma=0.058056 \mathrm{~m}^{-1}, L_{D}=3.7187 \mathrm{~m}$ and $k=0.7$.

$$
A=k \sqrt{\frac{2 p_{0}}{q_{0}}} \operatorname{sn}[t \exp (\sigma z)] \exp \left\{\left(\frac{\sigma}{2}+\gamma\right) z-\frac{i p_{0} e^{\sigma z}\left(1+k^{2}\right)}{\sigma}-\frac{i \sigma e^{\sigma z} t^{2}}{4 p_{0}}\right\}
$$

The amplitude and pulse width of these chirped periodic waves are given by

$$
\begin{aligned}
A & =k \sqrt{\frac{2 p_{0}}{q_{0}}} \frac{1}{\tau_{0}} \exp \left[\left(\frac{\sigma}{2}+\gamma\right) z\right], \\
\tau & =\tau_{0} \exp (-\sigma z) .
\end{aligned}
$$

The previous expressions imply that the intensity increases exponentially whereas the pulse width decreases exponentially. These variations clearly indicate that the three types of chirped waves do undergo compression in this tapered PCF. The compression of chirped periodic waves of sn, cn and dn types is depicted in Figs. 8, 9 and 10. In these figures, the dashed curves represent the intensity of the input chirped periodic waves and the solid curves correspond to the intensity of the compressed pulses. A train of USPs is thus possible using a tapered PCF for all the types of periodic waves.

\section{Case III: GVD Increases and Nonlinearity Decreases}

To meet with the above mentioned profiles, we reduce the diameter of air hole and keep the pitch a constant. A PCF length of $19.64 \mathrm{~m}$ which is two times the dispersion length is considered. In this 


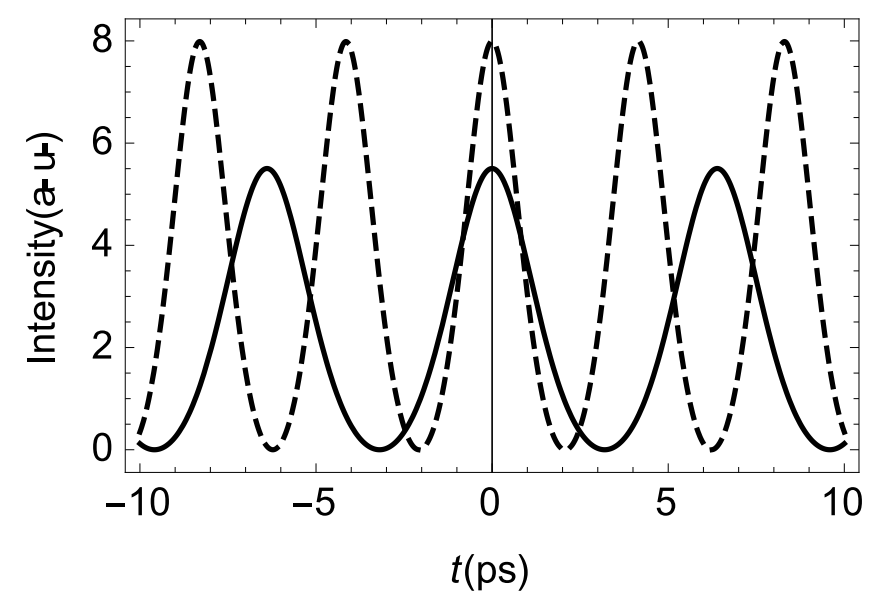

Figure 9.: Intensity profile of initial (dashed lines) and compressed output (solid lines) of the cnperiodic wave through a PCF. The physical parameters are $p_{0}=-0.0553713 \mathrm{ps}^{2} / \mathrm{m}, q_{0}=0.006794$ $\mathrm{W}^{-1} \mathrm{~m}^{-1}, \gamma=0.00398355 \mathrm{~m}^{-1}, \sigma=0.058056 \mathrm{~m}^{-1}, L_{D}=3.7187 \mathrm{~m}$ and $k=0.7$.

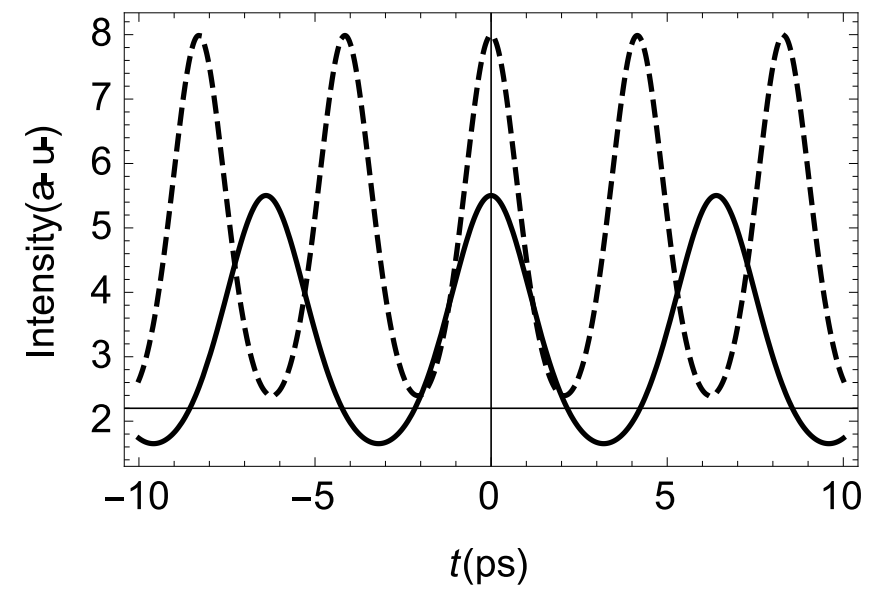

Figure 10.: Intensity profile of initial (dashed lines) and compressed output (solid lines) of the dnperiodic wave through a PCF. The physical parameters are $p_{0}=-0.0553713 \mathrm{ps}^{2} / \mathrm{m}, q_{0}=0.006794$ $\mathrm{W}^{-1} \mathrm{~m}^{-1}, \gamma=0.00398355 \mathrm{~m}^{-1}, \sigma=0.058056 \mathrm{~m}^{-1}, L_{D}=3.7187 \mathrm{~m}$ and $k=0.7$.

case, the relative air hole diameter is varied exponentially from 0.27537 to 0.39994 , the air hole diameter from 0.967 to $1.342 \mu \mathrm{m}$ and the pitch is kept at $3.35 \mu \mathrm{m}$. Figs. 11a and $11 \mathrm{~b}$ represent the variations of design parameters and optical properties against the propagation distance.

Now, we study the dynamics of the chirped waves in the designed tapered PCF wherein the dispersion increases and nonlinearity decreases. In this case the dn, cn and sn waves are,

$$
\begin{gathered}
A=\sqrt{\frac{2 p_{0}}{q_{0}}} d n[t \exp (-\sigma z)] \exp \left\{\left(-\frac{\sigma}{2}+\gamma\right) z-\frac{i p_{0} e^{-\sigma z}\left(2-k^{2}\right)}{\sigma}+\frac{i \sigma e^{-\sigma z} t^{2}}{4 p_{0}}\right\}, \\
A=k \sqrt{\frac{2 p_{0}}{q_{0}}} c n[t \exp (-\sigma z)] \exp \left\{\left(-\frac{\sigma}{2}+\gamma\right) z-\frac{i p_{0} e^{-\sigma z}\left(2 k^{2}-1\right)}{\sigma}+\frac{i \sigma e^{-\sigma z} t^{2}}{4 p_{0}}\right\}, \\
A=k \sqrt{\frac{2 p_{0}}{q_{0}}} \operatorname{sn}[t \exp (-\sigma z)] \exp \left\{\left(-\frac{\sigma}{2}+\gamma\right) z+\frac{i p_{0} e^{-\sigma z}\left(1+k^{2}\right)}{\sigma}+\frac{i \sigma e^{-\sigma z} t^{2}}{4 p_{0}}\right\} .
\end{gathered}
$$



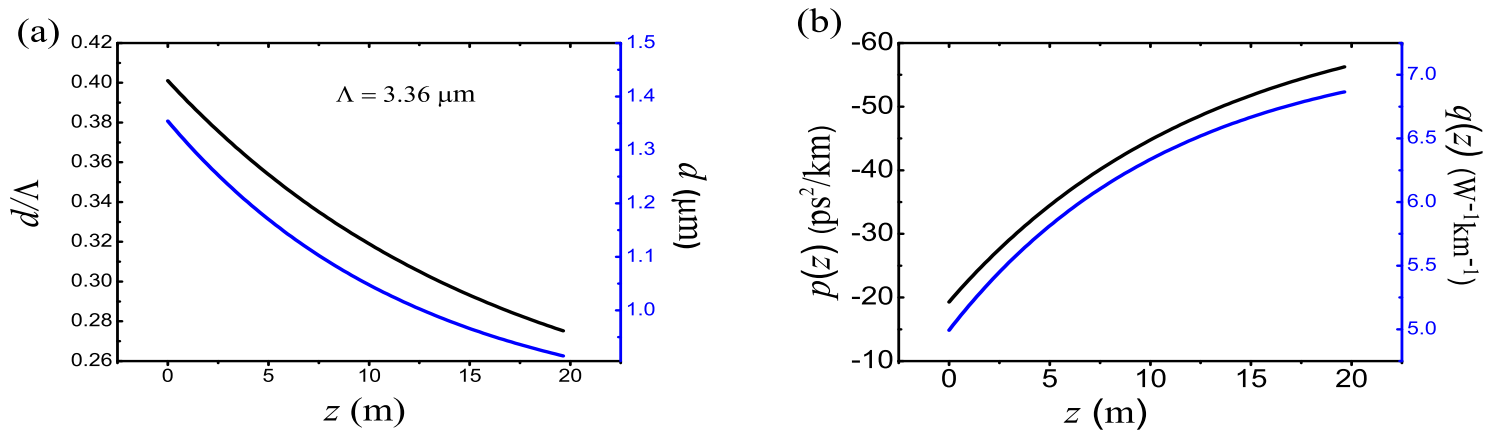

Figure 11.: (a) Variation of PCF design parameters $d / \Lambda$ and $d$ along the propagation distance, $z$. (b) Variation of GVD, $p(z)$, and nonlinearity, $q(z)$, as a function of propagation distance, $z$.

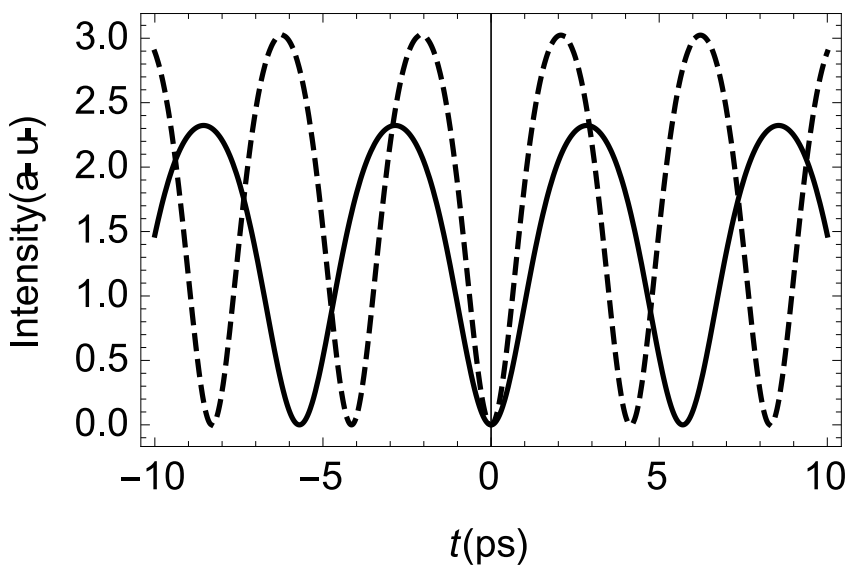

Figure 12.: Intensity profile of initial (dashed lines) and compressed output (solid lines) of the sn- periodic wave through a PCF. The physical parameters are $p_{0}=-0.020963 \mathrm{ps}^{2} / \mathrm{m}, q_{0}=$ $0.005112636 \mathrm{~W}^{-1} \mathrm{~m}^{-1}, \gamma=0.0013677738 \mathrm{~m}^{-1}, \sigma=0.0161538 \mathrm{~m}^{-1}, L_{D}=9.822 \mathrm{~m}$ and $k=0.7$.

The amplitude and pulse width of these chirped periodic waves are given by

$$
\begin{aligned}
A & =k \sqrt{\frac{2 p_{0}}{q_{0}}} \frac{1}{\tau_{0}} \exp \left(\left(-\frac{\sigma}{2}+\gamma\right) z\right) \\
\tau & =\tau_{0} e^{\sigma z}
\end{aligned}
$$

In contrast to the two previous cases, we find that the intensity decreases exponentially whereas the pulse width increases exponentially. This process clearly indicates that the three types of chirped waves undergo broadening in this tapered PCF. Figs. 12, 13 and 14 represent the dynamics of the three types of chirped waves. These waves undergo broadening when they propagate in this tapered PCF. The output waves which are stretched in the temporal domain are depicted as solid wave lines. Thus, this tapered PCF acts as a pulse stretcher which plays a vital role in the chirped pulse amplification.

\section{Case IV: Both GVD and Nonlinearity Increase}

In this case, a PCF of the same length as in the previous case is considered. We increase the relative air hole diameter from 0.39994 to 0.275367 and pitch from 1.342 to $0.964 \mu \mathrm{m}$ with the air hole diameter fixed at $3.35 \mu \mathrm{m}$. The corresponding variations are reflected in Figs. 15a and 15b, respectively.

The dn, cn and sn - waves for this case are, 


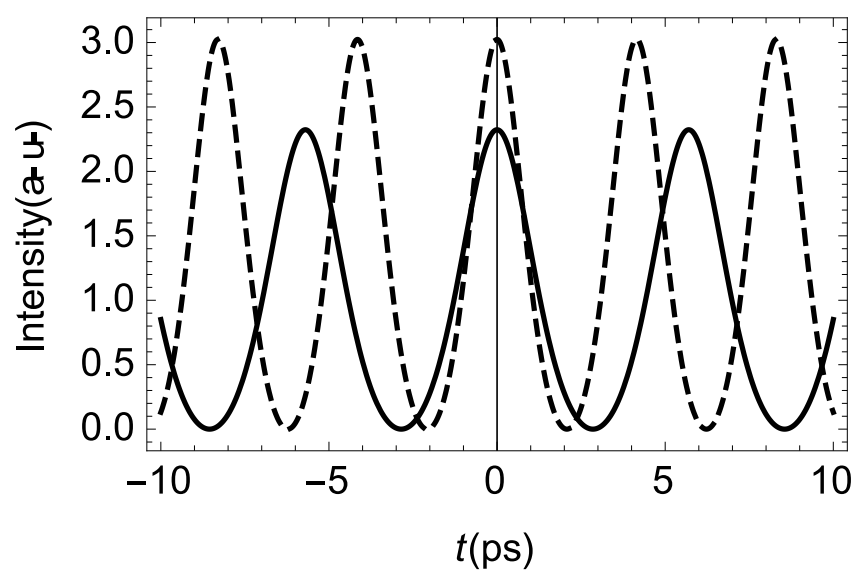

Figure 13.: Intensity profile of initial (dashed lines) and compressed output (solid lines) of the cn- periodic wave through a PCF. The physical parameters are $p_{0}=-0.020963 \mathrm{ps}^{2} / \mathrm{m}, q_{0}=$ $0.005112636 \mathrm{~W}^{-1} \mathrm{~m}^{-1}, \gamma=0.0013677738 \mathrm{~m}^{-1}, \sigma=0.0161538 \mathrm{~m}^{-1}, L_{D}=9.822 \mathrm{~m}$ and $k=0.7$.

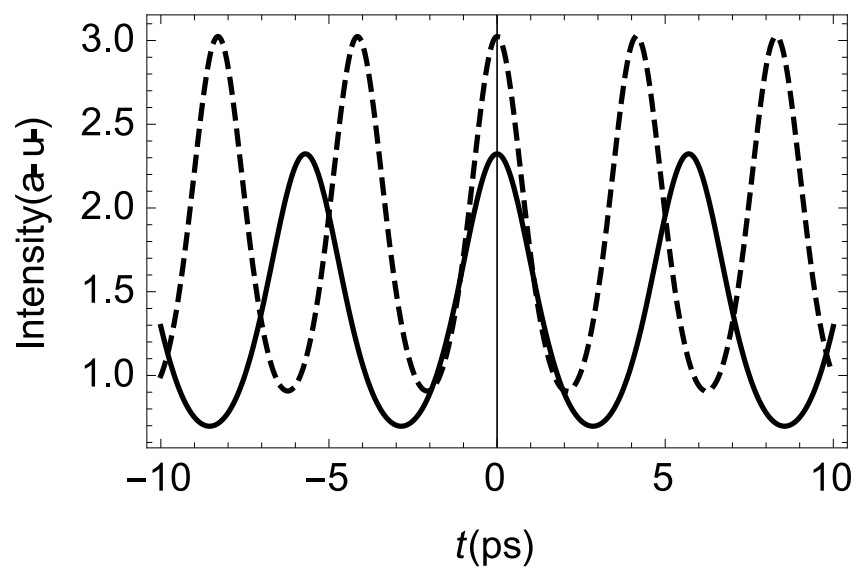

Figure 14.: Intensity profile of initial (dashed lines) and compressed output (solid lines) of the dn- periodic wave through a PCF. The physical parameters are $p_{0}=-0.0209631 \mathrm{ps}^{2} / \mathrm{m}, q_{0}=$ $0.005112636 \mathrm{~W}^{-1} \mathrm{~m}^{-1}, \gamma=0.0013677738 \mathrm{~m}^{-1}, \sigma=0.0161538 \mathrm{~m}^{-1}, L_{D}=9.822 \mathrm{~m}$ and $k=0.7$.

(a)

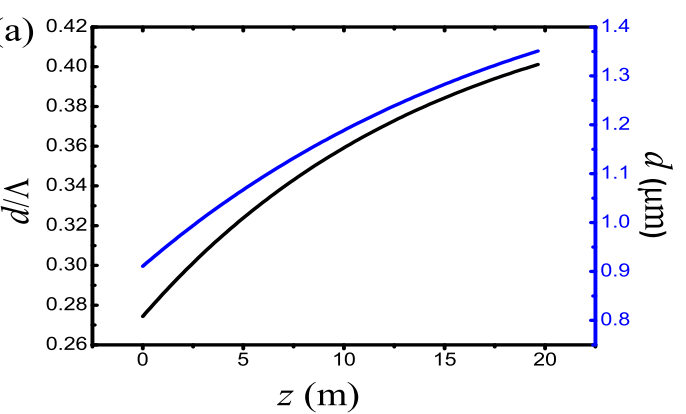

(b)

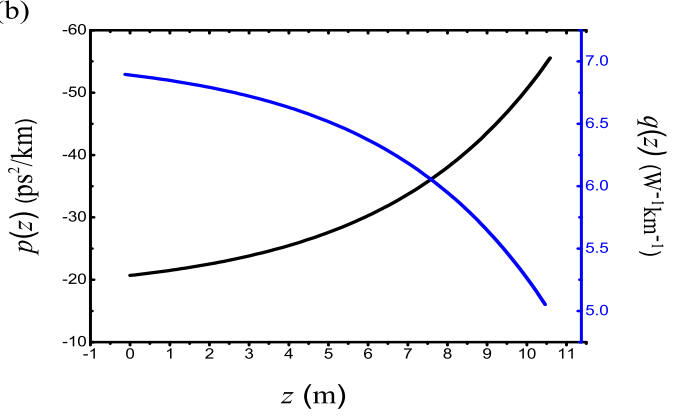

Figure 15.: (a) Variation of PCF design parameters $d / \Lambda$ and $d$ along the propagation distance, $z$.(b) Variation of GVD, $p(z)$, and nonlinearity, $q(z)$, as a function of propagation distance, $z$. 


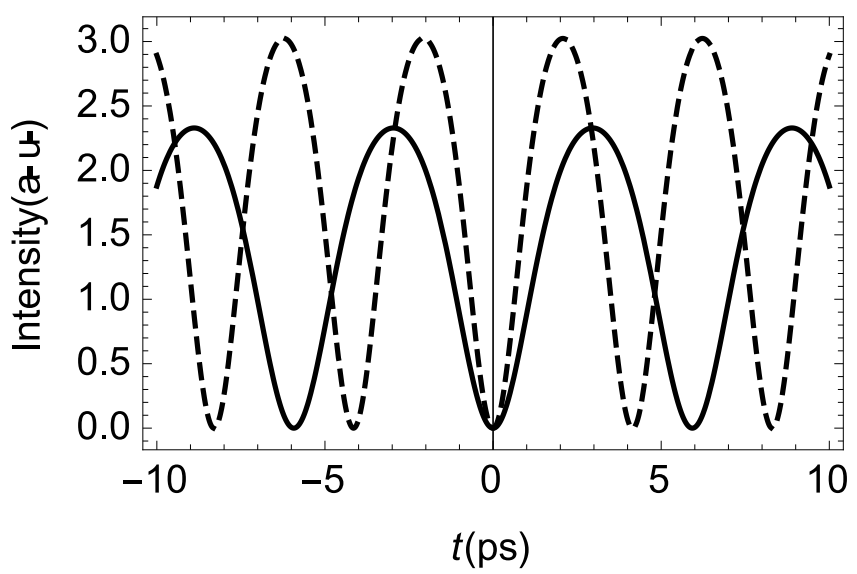

Figure 16.: Intensity profile of initial (dashed lines) and compressed output (solid lines) of the snperiodic wave through a PCF. The physical parameters are $p_{0}=-0.0209631 \mathrm{ps}^{2} / \mathrm{m}, q_{0}=0.006794$ $\mathrm{W}^{-1} \mathrm{~m}^{-1}, \gamma=0.002420401 \mathrm{~m}^{-1}, \sigma=0.01812684 \mathrm{~m}^{-1}, L_{D}=9.822 \mathrm{~m}$ and $k=0.7$.

$$
\begin{gathered}
A=\sqrt{\frac{2 p_{0}}{q_{0}}} d n[t \exp (-\sigma z)] \exp \left\{-\left(\frac{\sigma}{2}+\gamma\right) z-\frac{i p_{0} e^{-\sigma z}\left(2-k^{2}\right)}{\sigma}+\frac{i \sigma e^{-\sigma z} t^{2}}{4 p_{0}}\right\}, \\
A=k \sqrt{\frac{2 p_{0}}{q_{0}}} \operatorname{cn}[t \exp (-\sigma z)] \exp \left\{-\left(\frac{\sigma}{2}+\gamma\right) z-\frac{i p_{0} e^{-\sigma z}\left(2 k^{2}-1\right)}{\sigma}+\frac{i \sigma e^{-\sigma z} t^{2}}{4 p_{0}}\right\}, \\
A=k \sqrt{\frac{2 p_{0}}{q_{0}}} \operatorname{sn}[t \exp (-\sigma z)] \exp \left\{-\left(\frac{\sigma}{2}+\gamma\right) z+\frac{i p_{0} e^{-\sigma z}\left(1+k^{2}\right)}{\sigma}+\frac{i \sigma e^{-\sigma z} t^{2}}{4 p_{0}}\right\} .
\end{gathered}
$$

The amplitude and pulse width of the three types of chirped periodic waves are given by

$$
\begin{aligned}
A & =k \sqrt{\frac{2 p_{0}}{q_{0}}} \frac{1}{\tau_{0}} \exp \left(-\left(\frac{\sigma}{2}+\gamma\right) z\right), \\
\tau & =\tau_{0} e^{\sigma z} .
\end{aligned}
$$

Like in the previous case, this process also clearly signifies the broadening of the three types of chirped waves in this tapered PCF.

From Figs. 16, 17 and 18, one can observe the broadening of these chirped periodic waves when they propagate in this tapered PCF. Hence, this tapered PCF also can act as a pulse stretcher.

From these detailed analytical and numerical results, we corroborate that the PCF acts as a pulse compressor only when the dispersion decreases exponentially irrespective of the nonlinear profile variation. On the other hand, if the dispersion increases exponentially irrespective of the nonlinear profile variation, then the tapered PCF acts as a pulse stretcher which is an essential component in the chirped pulse amplification system. Thus, there is a freedom in choosing the type of medium based on the requirements. From the literature survey, it is very clear that the soliton based pulse compression has been studied widely in dispersion decreasing nonlinear media. However, there are a few pulse compression studies in dispersion increasing nonlinear media which are yet to be explored. Thus these physical conditions result in various optical media, which, in turn, open several avenues for generating the high quality USPs. 


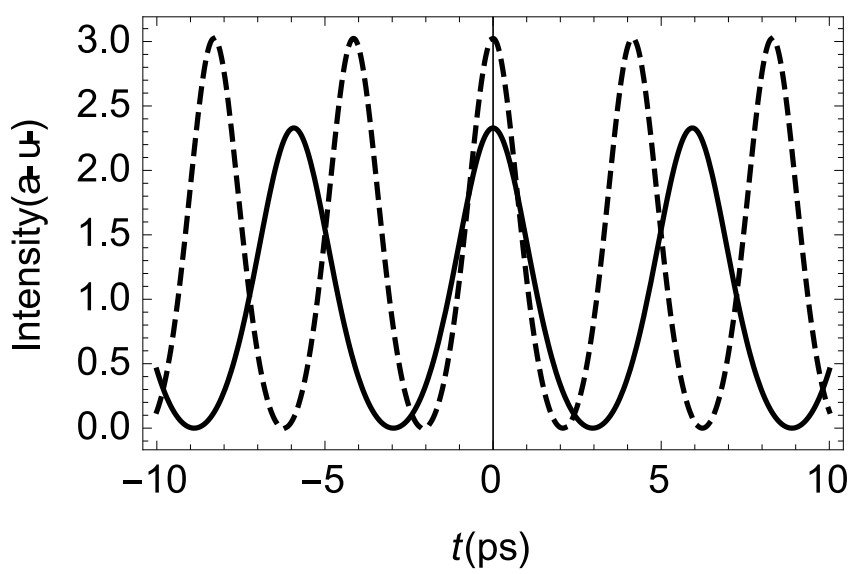

Figure 17.: Intensity profile of initial (dashed lines) and compressed output (solid lines) of the cnperiodic wave through a PCF. The physical parameters are $p_{0}=-0.0209631 \mathrm{ps}^{2} / \mathrm{m}, q_{0}=0.006794$ $\mathrm{W}^{-1} \mathrm{~m}^{-1}, \gamma=0.002420401 \mathrm{~m}^{-1}, \sigma=0.01812684 \mathrm{~m}^{-1}, L_{D}=9.822 \mathrm{~m}$ and $k=0.7$.

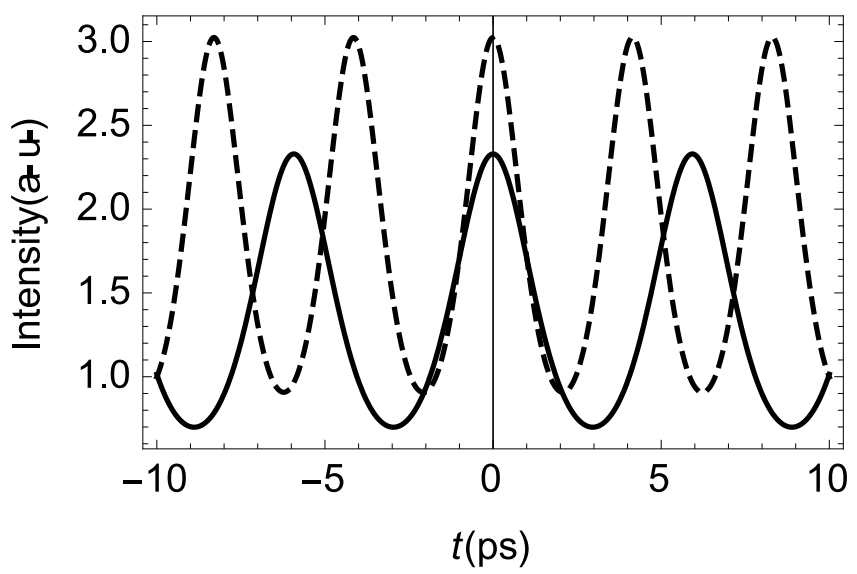

Figure 18.: Intensity profile of initial (dashed lines) and compressed output (solid lines) of the dnperiodic wave through a PCF. The physical parameters are $p_{0}=-0.0209631 \mathrm{ps}^{2} / \mathrm{m}, q_{0}=0.006794$ $\mathrm{W}^{-1} \mathrm{~m}^{-1}, \gamma=0.002420401 \mathrm{~m}^{-1}, \sigma=0.01812684 \mathrm{~m}^{-1}, L_{D}=9.822 \mathrm{~m}$ and $k=0.7$.

At this juncture, we intend to record that the stability of the compressed pulses has already been reported in detail [30]. That is, the robustness of the proposed silica core PCF pulse compressor has already been thoroughly studied by considering various perturbations such as variation in loss coefficient (increasing and decreasing values of loss) for a single pulse as well as a train of pulses of hyperbolic and raised-cosine profiles. In order to evaluate the robustness of the proposed compressors, deviations in the pedestal energy and nal compression factor have been computed. It has been found that the train of pulses are stable against the perturbations. Based on this previous experience, we anticipate that the train of pulses studied in this paper may also be stable against perturbations.

Very recently, high power pulse trains excited by modulated continuous waves have been investigated [31] wherein pulse trains were theoretically demonstrated under the influence of higher order linear and nonlinear effects. However, in this paper, the pulse trains have been achieved by various chirped periodic waves of sn, cn and dn types, which are the exact solutions of the governing equation. Further, in Ref.[31], the pulse train was discussed in the homogeneous fiber medium wherein the dispersion and nonlinear properties do not vary along the propagation direction. On the other hand, in this paper, the trains of short pulses have been achieved owing to variation 


\section{of above mentioned optical properties along the propagation direction.}

\section{Dynamics of Soliton: Long Wave Limit}

It is obvious that the periodic waves are converted into solitary type pulses under long wave limit. We also discuss the formation of chirped bright solitary pulse for Eq. (1) at the long wave limit. When $k \rightarrow 1$ and $\mathrm{cn}$, dn $\rightarrow$ sech, we get the following solitary type pulse from Eqs. (20) and (21).

$$
A=\sqrt{\frac{2 p_{0}}{q_{0}}} \operatorname{sech}[t \exp (\sigma z)] \exp \left\{\left(\frac{\sigma}{2}-\gamma\right) z+\frac{i p_{0} e^{\sigma z}}{\sigma}-\frac{i \sigma e^{\sigma z} t^{2}}{4 p_{0}}\right\} .
$$

Similarly, the dark soliton is obtained from Eq. (22) when $k \rightarrow 1$ and $s n \rightarrow$ tanh,

$$
A=\sqrt{\frac{2 p_{0}}{q_{0}}} \tanh [t \exp (\sigma z)] \exp \left\{\left(\frac{\sigma}{2}-\gamma\right) z-\frac{2 i p_{0} e^{\sigma z}}{\sigma}-\frac{i \sigma e^{\sigma z} t^{2}}{4 p_{0}}\right\}
$$

Here, we emphasize that the USPs can also be generated with the above discussed solitary type pulses. However, this is beyond scope of the present study.

\section{Chirped Periodic Waves by Self-Similar Analysis}

In order to compare the chirped periodic waves derived by HBT method, we also show the generation of chirped periodic waves using the self-similar analysis. We adopt the procedure developed by Kruglov et al [32]. According to the self-similar analysis, the complex function $A(z, t)$ can be expressed as,

$$
A(z, t)=U(z, t) \exp [i \phi(z, t)]
$$

where $U$ and $\phi$ which are real functions of $z$ and $t$ represent the amplitude and phase, respectively. We assume that the amplitude of the self-similar solutions of Eq. (1) is given by,

$$
U(z, t)=\frac{1}{\sqrt{1+\alpha_{20} D(z)}} R\left[\frac{t-t_{c}}{1+\alpha_{20} D(z)}\right] \exp [G(z)]
$$

The phase is given as,

$$
\phi=\alpha_{1}(z)+\alpha_{2}(z)\left(t-t_{c}\right)^{2}
$$

where $t_{c}, D(z)$ and $G(z)$ are the center of the pulse, cumulative dispersion and cumulative loss/gain, respectively. The constant phase and chirp parameters are represented by $\alpha_{1}$ and $\alpha_{2}$, respectively. $R$ is a function to be identified. The functions $D(z), G(z), \alpha_{1}$ and $\alpha_{2}$ are defined by

$$
\begin{aligned}
& D(z)=4 \int_{0}^{z} p\left(z^{\prime}\right) d z^{\prime} \\
& G(z)=\int_{0}^{z} g\left(z^{\prime}\right) d z^{\prime}
\end{aligned}
$$




$$
\alpha_{1}(z)=\alpha_{10}+\lambda \int_{0}^{z} \frac{p\left(z^{\prime}\right)}{\left(1+\alpha_{20} D\left(z^{\prime}\right)\right)^{2}} d z^{\prime}
$$

and

$$
\alpha_{2}(z)=\frac{\alpha_{20}}{1+\alpha_{20} D(z)}
$$

The parameters $\lambda, \alpha_{10}$ and $\alpha_{20}$ are the constants of integration. It is useful to define $\rho(z)$, which is the ratio of the dispersion to the nonlinearity given by,

$$
\rho(z)=\rho(0)\left(1+\alpha_{20} D(z)\right) \exp (2 G(z))=\frac{p(z)}{q(z)} .
$$

Differentiating Eq. (49) gives,

$$
\gamma(z)=\frac{1}{2 \rho(z)} \frac{d \rho}{d z}-\frac{2 \alpha_{20} p(z)}{1+\alpha_{20} D(z)}
$$

Further, Eq. (50) provides the necessary and sufficient condition for various functions $p(z), q(z)$ and $\gamma(z)$ for the existence of self-similar solutions of the NLSE. Finally, the self-similar solution for exponentially decreasing dispersion and exponentially increasing nonlinearity is given by,

$$
A=\sqrt{\frac{2 p_{0} \exp (-\sigma z)}{q_{0} \exp (2 \gamma z)}} \frac{1}{T_{0} \exp (-\sigma z)} d n\left(\frac{t-t_{c}}{T_{0} \exp (-\sigma z)}\right) \exp \left\{i \alpha_{10}+\frac{i p_{0}\left(e^{\sigma z}-1\right)\left(2-k^{2}\right)}{\sigma T_{0}^{2}}-\frac{i \sigma e^{\sigma z}\left(t-t_{c}\right)^{2}}{4 p_{0}}\right\},
$$

where $T_{0}$ and $\alpha_{20}$ represent the initial pulse width and chirp, respectively. It is also observed that the coefficient of the loss/gain term has to be same as the decay (growth) rate of the nonlinearity. Similarly, one can also derive the other forms of periodic waves (cn and sn) for the above mentioned exponential variations in the fiber medium. Further, we intend to report that these periodic waves can also be theoretically generated for the other possible exponential variations of fiber media. As a final note, we show that chirped periodic wave solution Eq. (20) derived by HBT method does match with that of the one obtained based on the self-similar analysis Eq. (51).

\section{Conclusions}

We have considered wave propagation in a tapered photonic crystal fibre wherein the dispersion, nonlinearity and gain/loss vary along the propagation direction. The wave propagation is governed by a variable coefficient NLSE. We have discussed the formation of chirped periodic waves of sn, cn and dn types by solving the NLSE using Hirota bilinear method. Further, we have found that these chirped periodic waves exist not only in a medium with exponentially decreasing dispersion and exponentially increasing nonlinearity, but also in other media with exponential variations of dispersion and nonlinearity. Based on these analytical results, we have designed four different types of tapered PCFs. We have dealt with the generation of a train of USPs using these chirped periodic waves in various tapered PCFs. From the detailed analysis, it has been found that exponentially decreasing dispersion PCFs (case I and II) act as compressors whereas the exponentially increasing dispersion PCFs (case III and IV) act as stretchers. Under the long wave limit, chirped solitary type pulses have also been derived from the chirped periodic waves. Finally, the chirped periodic waves obtained by Hirota bilinear method have been compared with that obtained by using self-similar analysis. We envisage that the newly designed four types of tapered PCFs would be highly useful in the USP generation and also in the chirped pulse amplification as a stretcher. 
Acknowledgements: SOA wishes to thank the Ministry of Education , Nigeria for financial support through the TETFUND scholarship scheme. KSN wishes to thank CSIR [No: 03(1264)/12/EMR-II], Government of India, for the financial support through the project.

\section{References}

[1] Hasegawa, A.; Tappert, F. Applied Physics Letters 1973, 23 (3), 142-144.

[2] Hasegawa, A.; Kodama, Y. Solitons in optical communications.7 : , 1995.

[3] Mollenauer, L.F.; Stolen, R.H.; Gordon, J.P. Phys. Rev. Lett. 1980, 45 (13), 1095.

[4] Mollenauer, L.F.; Gordon, J.P. Solitons in optical fibers: fundamentals and applications. : , 2006.

[5] Agrawal, G. Applications of nonlinear fiber optics. : , 2001.

[6] Hasegawa, A. Opt. Lett. 1984, 9 (7), 288-290.

[7] Siders, C.W.; Siders, J.L.; Taylor, A.J.; Park, S.G.; et al. Appl. Opt. 1998, 37 (22), 5302-5305.

[8] Robinson, T.; O'Keeffe, K.; Landreman, M.; Hooker, S.; Zepf, M.; et al. Opt. Lett. 2007, 32 (15), $2203-2205$.

[9] Shank, C.; Fork, R.; Yen, R.; Stolen, R.; et al. Appl. Phys. Lett. 1982, 40 (9), 761-763.

[10] Travers, J.; Stone, J.M.; Rulkov, A.; Cumberland, B.; George, A.; Popov, S.; Knight, J.; et al. Opt. Express 2007, 15 (20), 13203-13211.

[11] Colman, P.; Husko, C.; Combrié, S.; Sagnes, I.; Wong, C.; et al. Nature Photonics 2010, 4 (12), 862-868.

[12] Li, Q.; Wai, P.; Senthilnathan, K.; et al. J. Lightwave Technol. 2011, 29 (9), 1293-1305.

[13] Olupitan, S.; Senthilnathan, K.; Babu, P.R.; Aphale, S.S.; et al. Photonics Journal, IEEE 2012, 4 (5), $1420-1437$.

[14] C. Mak, C.; W. Chow, K.; Nakkeeran, K. J. Phys. Soc. Japan 2005, 74 (5), 1449-1456.

[15] Agrawal, G.P. Nonlinear fiber optics. : , 2013.

[16] Akhmediev, N.; Eleonskii, V.; Kulagin, N. Theoretical and mathematical physics 1987, 72 (2), 809-818.

[17] Serkin, V.N.; Hasegawa, A. Phys. Rev. Lett. 2000, 85 (21), 4502.

[18] Nakkeeran, K. Nonlinearity 2002, 15 (6), 1747-1754.

[19] Nakkeeran, K. J. Phys. A: Mathematical and General 2001, 34 (24), 5111.

[20] Burtsev, S.; Zakharov, V.E.; Mikhailov, A.V. Theoretical and Mathematical Physics 1987, 70 (3), $227-$ 240.

[21] Balakrishnan, R. Phys. Rev. A: At., Mol., Opt. Phys. 1985, 32 (2), 1144.

[22] Xu, Z.; Li, L.; Li, Z.; Zhou, G.; et al. Phys. Rev. E: Stat., Nonlinear, Soft Matter Phys. 2003, 68 (4), 046605 .

[23] Hirota, R. The direct method in soliton theory. In: ; Vol. 155, : , 2004.

[24] Wing Chow, K.; Kit Lam, C.; Nakkeeran, K.; et al. J. Phys. Soc. Japan 2008, 77 (5), 054001.

[25] Aranson, I.S.; Kramer, L. R. Mod. Phys. 2002, 74 (1), 99.

[26] Nozaki, K.; Bekki, N. J. Phys. Soc. Japan 1984, 53, 1581.

[27] Serkin, V.N.; Hasegawa, A. Selected Topics in Quantum Electronics, IEEE Journal of 2002, 8 (3), 418-431.

[28] Özemir, C.; Güngör, F. Reviews in Mathematical Physics 2012, 24 (07), 1250015.

[29] Lawden, D.F. Elliptic functions and applications. In: ; Vol. 80, : , 2013.

[30] Olupitan, S.; Senthilnathan, K.; Ramesh Babu, P.; Vasantha Jayakantha Raja, R.; Aphale, S.S.; et al. Journal of Modern Optics 2013, 60 (5), 368-377.

[31] Wang, Y.; Song, L.; Li, L.; et al. JOSA B 2015, 32 (11), 2257-2263.

[32] Kruglov, V.; Peacock, A.; Harvey, J. Phys. Rev. Lett. 2003, 90 (11), 113902. 